\title{
POLARIZATION DURING CAUSTIC CROSSING
}

\author{
ERIC AGOL \\ Physics Department \\ University of California, Santa Barbara \\ Santa Barbara, CA 93106
}

\begin{abstract}
The limbs of hot stars are polarized due to electron scattering, but this polarization cancels out due to rotational symmetry. During microlensing, the star is amplified by different amounts across its surface so that the limb polarization no longer cancels out, but can be observable. Polarization can be much higher for a caustic crossing during microlensing by a binary lens than for a single lens.
\end{abstract}

\section{Introduction}

Measuring polarization during binary microlensing can be useful in a number of ways: 1) It will allow another test of stellar atmosphere theory. 2) It can be used to confirm that flux variations are due to microlensing and not due to other variable star phenomena (such as "bumper stars"). 3) It can determine the Einstein radius of the lens. 4) The polarization angle gives the direction of the velocity and the position angle of the binary lens on the sky.

\section{Results}

Figure 1 shows the amplification and percent polarization during a typical caustic crossing for stars of various radii relative to the Einstein radius. These results assume a pure electron scattering atmosphere and that the caustic is straight and the star is moving perpendicular to the caustic. The same parameters are used as in Schneider and Weiß (1986), their figure $9 \mathrm{a}$. The polarization can be as large as $1 \%$ and the polarization angle flips twice as the caustic amplifies different parts of the star. The peak polarization is larger for smaller stars since the part of the star inside the caustic is more highly amplified than for a large star. This is the opposite dependence of polarization for a single lens, in which the larger stars are 


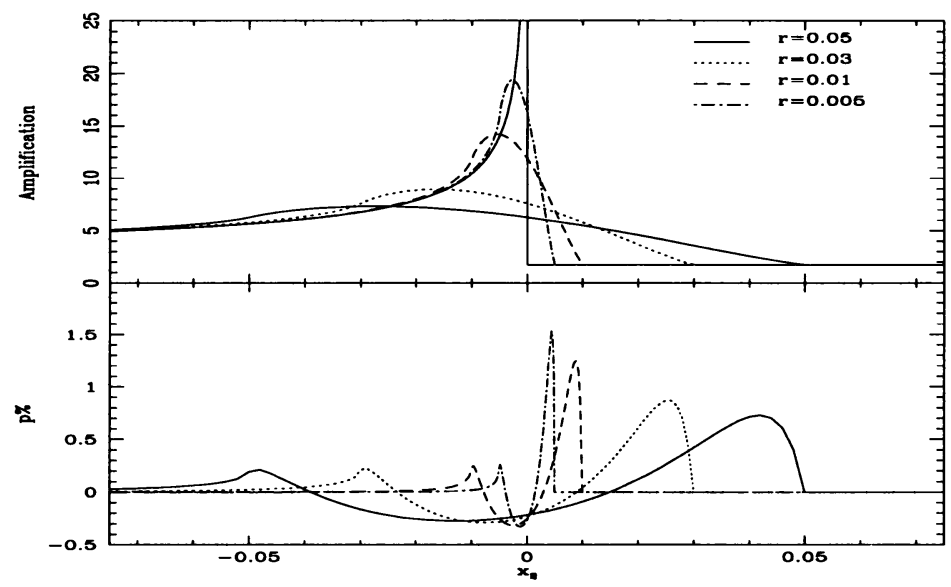

Figure 1. Amplification and percent polarization as a star crosses a caustic. The position of the center of the star is on the $x$ axis for various $r$, which is the ratio of the radius of the star to the Einstein radius of the lens in the source plane.

polarized more since the change in amplification across the star is larger. The amplitude of the polarization and the separation of the zero crossings both are determined by the size of the star relative to the Einstein radius, so if the stellar radius is known (e.g. spectroscopically), then the Einstein radius of the lens can be determined from the polarization light curve. This can be compared with the size determined from the amplification light curve to see if there are other effects on the polarization, such as absorption.

Polarization is highest for hot stars ( $\mathrm{O}$ and $\mathrm{B}$ ) since electron scattering dominates the opacity in their atmospheres at certain wavelengths (especially just above the Lyman and Balmer edges). For these stars, a typical ratio of the stellar radius to Einstein radius is $0.005-0.02$. There are no 0 and $B$ stars in the Galactic bulge, so observations will have to be done towards the LMC or M31.

A caustic crossing will typically last for $\sim 10$ hours which is long enough to be measurable with a large telescope for hot stars in the LMC. For a $\mathrm{B}=18$ star observed for 100 minutes with a 4 meter telescope (assuming $10 \%$ efficiency), the polarization error is $0.05 \%$.

More details on the calculations and further results can be found in Agol (1995).

\section{References}

Agol, E. 1995, MNRAS, submitted

Schneider, P., and Weiss, A., 1986, A\&A, 164, 237 